\title{
Traditionelle chinesische Arzneitherapie bei Patienten mit chronischer Rhinosinusitis - eine Therapiebeobachtung mit Berücksichtigung der Arzneimittelherkunft
}

\author{
Josef Hummelsberger ${ }^{a}$ Fritz Friedl $^{b}$ Wilhelm Gaus ${ }^{c}$ Ralph Kohnen ${ }^{d}$ Heidi Heuberger ${ }^{e}$ \\ Rebecca Seidenberger ${ }^{e} \quad$ Pamela Aidelsburger $^{f}$ Rudolf Bauer $^{g} \quad$ Günther Heubl $^{\mathrm{h}}$
}

a Societas Medicinae Sinensis, München, Deutschland; ${ }^{\mathrm{b}}$ Klinik Silima, Riedering, Deutschland; ${ }^{\mathrm{c}}$ Universität Ulm, Medizinische Fakultät, Institut für Epidemiologie und Medizinische Biometrie, Ulm, Deutschland; ${ }^{d}$ RPS Research Germany GmbH, Nürnberg, Deutschland;

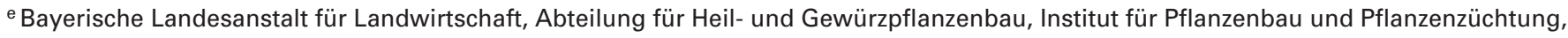
Freising, Deutschland; ${ }^{f}$ Consulting and Research in Health Economics and Medical Management (CAREM) GmbH, Sauerlach, Deutschland; $\mathrm{g}$ Institut für Pharmazeutische Wissenschaften, Karl-Franzens-Universität Graz, Graz, Österreich; ${ }^{\mathrm{h}}$ Ludwig-Maximilians-Universität München, Fakultät für Biologie, Systematische Botanik und Mykologie, München, Deutschland

\section{Schlüsselwörter}

Chinesische Arzneimittel . Anbau chinesischer Heilpflanzen · TCM .

Chronische Rhinosinusitis · Therapiebeobachtung

\section{Zusammenfassung}

Hintergrund: Chinesische Arzneimittel werden in Deutschland zunehmend angewandt. Der Import erfolgt primär aus China, mit der Folge einer erschwerten Qualitätskontrolle und Gewährleistung der Therapiesicherheit. Aus diesem Grund werden wichtige chinesische Arzneipflanzen seit 1999 in einem interdisziplinären Forschungsprojekt von der Bayerischen Landesanstalt für Landwirtschaft kontrolliert angebaut und wissenschaftlich erforscht, mit inzwischen bereits 16 "bayerischen Arzneidrogen", die in der lokalen Versorgung verwendet und über Apotheken abgegeben werden. Trotz vergleichbarer Qualität gibt es Vorbehalte, denn die bayerischen Arzneimitte werden seit 2006 in Deutschland eingesetzt, ohne dass die Wirksamkeit im Vergleich zu den chinesischen Arzneimitteln untersucht wurde. Aus diesem Grund führten wir eine Therapiebeobachtung mit einem Parallelgruppendesign bei Patienten mit chronischer Rhinosinusitis durch. Methodik: Die Beobachtungsdauer betrug 4 Wochen. Nach einem 4-wöchigen Follow-up wurden die Patienten nochmals telefonisch befragt. Während der Therapiebeobachtung nahmen die Patienten täglich $2 \times 50 \mathrm{ml}$ eines Dekokts aus chinesischen Arzneimitteln entweder (a) überwiegend aus bayerischem kontrolliertem Versuchsanbau (Bayern-Gruppe) oder (b) rein chinesischer Handelsware (China-Gruppe) zu sich. Die Bewertung des Therapieerfolgs erfolgte durch Numerische Rating-Skalen. Ergebnisse: Insgesamt beendeten 64 Patienten die Therapiebeobachtung (31 Bayern-Gruppe, 33 ChinaGruppe). In beiden Gruppen zeigten sich deutliche Verbesserungen in den Hauptsymptomen der chronischen Rhinosinusitis wie auch in den Nebensymptomen wie Infektneigung und allgemeiner Gesundheitszustand. Zwischen den Gruppen gab es keine relevanten Unterschiede bei der Besserung der Hauptsymptome. Die Dekokte waren insgesamt gut verträglich, schwere Nebenwirkungen traten nicht auf. Schlussfolgerung: Chinesische Arzneimittel aus bayerischem Anbau waren in dieser Beobachtungsstudie ähnlich wirksam wie Arzneikräuter aus China, allerdings müssen auch die Effekte der Begleittherapie, insbesondere der Akupunktur berücksichtigt werden. Die Veränderung der Symptome während der Behandlungszeit sollte zu weiteren Studien Anlass geben, die die Wirksamkeit dieser Arzneimittelrezeptur bei chronischer Rhinosinusitis untersuchen.

(C) 2015 S. Karger GmbH, Freiburg

\section{Keywords}

Chinese herbal medicine - Chinese plants cultivation - TCM .

Chronic rhinosinusitis - Observational trial

\section{Summary}

Traditional Chinese Pharmacotherapy in Patients with Chronic Rhinosinusitis - an Observational Trial Considering the Origin of Medicinal Drugs

Background: The use of Chinese medicinal drugs is becoming more common in Germany. However, the import from China results in aggravated quality controls and potentially jeopardized therapeutic safety. Therefore, in 1999 the Bavarian Department for Agriculture has initiated an interdisciplinary research project to cultivate and analyze important Chinese herbal plants. Currently 16 Bavarian-produced Chinese drugs are in use and distributed to patients by pharmacies. Despite a comparable quality of Bavarian pharmaceutical products, there are concerns remaining as the Bavarian medical drugs have been used for treatment purposes on patients since 2006, without their effect having been compared to the Chinese products. Therefore we performed an observational trial using a parallel group design on patients with chronic rhinosinusitis. Methods: The duration of the trial was 4 weeks. After a 4 -week follow-up, the patients were interviewed via telephone. During the trial the patients were given $2 \times$ $50 \mathrm{ml}$ of a decoction of Chinese medicinal herbs, either (a) from Bavarian controlled cultivation (Bavaria group) or (b) from Chinese production (China group). The therapeutic success was evaluated using numerical rating scales. Results: In total, 64 patients completed the observational trial (31 Bavaria group, 33 China group). Both groups showed significant improvements in the main symptom scores of chronic rhinosinusitis as well as in secondary symptoms, such as the overall state of health or the tendency to catch a cold. There were no significant differences between the groups concerning the main symptoms scores. Overall the herbal decoctions had no severe side effects. Conclusion: This observational trial shows that Chinese herbal drugs from Bavarian cultivation are as effective as medicinal herbs imported from China, but the effects of concomitant therapies must be considered as well. The symptom score improvements during the treatment period were obvious and should stimulate further investigation on the efficacy of this herbal formula in the treatment of chronic rhinosinusitis.

\section{KARGER}

(c) 2015 S. Karger GmbH, Freiburg

$1661-4119 / 15 / 0225-0312 \$ 39.50 / 0$ 


\section{Hintergrund}

Chinesische Arzneimittel werden in Deutschland zunehmend häufiger verwendet. In einer repräsentativen Interviewstudie aus dem Jahre 2002 [1] gaben 1,6\% der Befragten an, in den vergangenen 12 Monaten chinesische Arzneimittel (explizit ohne Akupunktur) verwendet zu haben. Die Therapie erfolgt meist in Form von Dekokten individualisierter Magistral-Rezepturen aus 5-12 pflanzlichen Arzneidrogen, die entsprechend den Prinzipien der traditionellen chinesischen Medizin (TCM) zusammengestellt werden. Häufig kommen begleitend weitere Therapieformen wie Akupunktur, manuelle Techniken (Tuina) und diätetische Beratungen zum Einsatz.

Der Import der Arzneidrogen, primär aus China, erschwert die Qualitätskontrolle und die Gewährleistung der Therapiesicherheit. So kommt es bei den importierten Drogen immer wieder zu Problemen bezüglich der Identifikation [2] sowie zu Belastungen mit Schwermetallen, Pestiziden oder Aflatoxinen. Die Qualitätskontrollen durch Importeure und Apotheken in Deutschland gewährleisten, dass gefährdende Drogen nicht verwendet werden und die Arzneimittelsicherheit gegeben ist [3]. Dennoch ist ein kontrollierter Anbau von häufig verwendeten chinesischen Arzneipflanzen in Deutschland ein wichtiger und sinnvoller Ansatz.

In einem vom Bayerischen Landwirtschaftsministerium finanziell geförderten interdisziplinären Projekt mit mehreren Projektpartnern werden aktuell 22 ausgewählte chinesische Heilpflanzenarten systematisch erforscht und so Anbauanleitungen erstellt [4]. Auf dieser Basis werden von Landwirten, die auf Arzneipflanzen spezialisiert sind, bereits «bayerische Drogen» erzeugt, in der Versorgung verwendet und über Apotheken abgegeben. Die aktuelle Praxis deutet darauf hin, dass die Entscheidung für eine Verwendung chinesischer Arzneikräuter aus bayerischem Anbau willkürlich auf der Basis von Verfügbarkeit oder persönlichen Vorlieben erfolgt. Auch wurden wiederholt Vorbehalte gegenüber der Qualität und Wirksamkeit der Arzneidrogen aus bayerischem Anbau geäußert. Gleichzeitig zeigen Wirkstoff-, sensorische und Qualitätsanalysen eine hohe, vergleichbare Qualität der bayerischen Arzneidrogen [5].

Aus wissenschaftlicher Sicht wäre es sinnvoll, die Wirksamkeit der bayerischen Drogen gleichzeitig mit Placebo und chinesischen Drogen im Rahmen randomisierter kontrollierter Studien zu vergleichen. Unter den derzeit in Deutschland gültigen Rahmenbedingungen ist die Durchführung derartiger Studien jedoch nur erschwert möglich, da umfangreiche Voraussetzungen für die Genehmigung gegeben sein müssen. Die hier für die Therapie verwendeten chinesischen Arzneimittelrezepturen werden individuell zusammengestellt. Ein Herstellerinteresse, die notwendigen Vorarbeiten für eine formale klinische Prüfung zu erbringen, ist deshalb nicht vorhanden. Aus diesem Grund wurde als Studiendesign eine vergleichende Therapiebeobachtung gewählt [6-9]. Bei einer Therapiebeobachtung wird nicht in die Therapieentscheidungen des Arztes eingegriffen, die Praxissituation wird unverändert abgebildet und dokumentiert. Dennoch sind vergleichende Aussagen zu einfachen Fragestellungen möglich.
Diese Therapiebeobachtung wurde zum in der Praxis häufigen und wichtigen Indikationsbereich der chronischen Rhinosinusitis durchgeführt.

Die Fragestellungen und Vorgaben waren:

- Anwendung der verwendeten Rezeptur, Modifikationen und andere Therapien durch die beteiligten Ärzte.

- Ergeben sich aus der Bewertung durch den Patienten mit chronischer Rhinosinusitis oder behandelnden Arzt Unterschiede in der Wirksamkeit zwischen einem Dekokt aus original chinesischen Kräutern und einem Dekokt aus bayerischem Anbau?

- Ergeben sich aus der Bewertung durch den Patienten mit chronischer Rhinosinusitis Unterschiede in der Verbesserung einzelner Symptome zwischen einem Dekokt aus original chinesischen Kräutern oder einem Dekokt aus bayerischem Anbau?

- Ergeben sich aus der Bewertung durch den Patienten mit chronischer Rhinosinusitis Unterschiede in der Verträglichkeit zwischen einem Dekokt aus original chinesischen Kräutern und einem Dekokt aus bayerischem Anbau?

- Unterscheiden sich Häufigkeit und Schwere von unerwünschten Therapiewirkungen bei Einnahme eines Dekokts aus original chinesischen Kräutern und eines Dekokts aus bayerischem Anbau?

\section{Methodik}

Diese Pilotstudie wurde als prospektive, multizentrische (10 Ärzte mit mindestens $350 \mathrm{~h}$ Ausbildung in chinesischer Medizin und Phytotherapie-Prüfung im Bereich chinesische Arzneitherapie) und nicht-interventionelle vergleichende Therapiebeobachtung durchgeführt, ohne Eingriff in die Therapieentscheidungen der teilnehmenden Ärzte.

Die Beobachtungsdauer pro Patient betrug 4 Wochen. Vor der Therapie sowie 2 und 4 Wochen danach füllten die Patienten in den Arztpraxen die Fragebögen aus. Vier Wochen nach Ende der Therapiebeobachtung wurden die Patienten nochmals telefonisch von der Studienmitarbeiterin befragt.

Während der Therapiebeobachtung nahmen die Patienten täglich $2 \times$ $50 \mathrm{ml}$ eines Dekokts aus chinesischen Arzneimitteln, entweder überwiegend aus bayerischem kontrollierten Anbau oder rein chinesischer Handelsware zu sich. Die Zusammensetzung des Dekokts wurde in einem umfangreichen Expertenprozess festgelegt und zielte auf die im Sinne der TCM wichtigen Syndrommuster «Wind-Kälte» (algor venti, fenghan), energetische Schwäche des Qi des Fk Lunge (depletio qi pulmonalis, feiqi xu), «Schleim» (pituita, tan) und «Hitze» (calor, re). Die Formel wurde gemäß den klassischen Rezepten «Fk Lunge (o. pulmonalis) erwärmende und den (Nasen-)Fluss stoppende Pille» (Wenfei zhiliu wan), "Pulver gegen Wind aus Jade» (Yu pingfeng san) und

Basisrezeptur bei chronischer Rhinosinusitis

Angelica dahurica radix (Baizhi) $6 \mathrm{~g}$

Saposhnikoviae radix (Fangfeng) $3 \mathrm{~g}$

Astragali radix (Huangqi) $6 \mathrm{~g}$

Scutellariae radix (Huangqin) $3 \mathrm{~g}$

Atractylodis macrocephalae rhizoma (Baizhu) $6 \mathrm{~g}$

Platycodi radix (Jiegeng) $6 \mathrm{~g}$

Pinelliae rhizoma (Banxia) $3 \mathrm{~g}$

Magnoliae flos (Xinyihua) $1 \mathrm{~g}$

Coicis semen (Yiren) $9 \mathrm{~g}$

Glycyrrhizae radix (Gancao) $2 \mathrm{~g}$ 
«Xanthium-Pulver» (Cang'erzi san) erstellt und orientierte sich zudem an einer Studie zur modernen Rezeptur «Sinusolve» [10] (Kasten 1): Das Rezept bestand zum entscheidenden Teil (so genannte Haupt- und Minister-Arzneien) aus Kräutern, die auch als Apothekenware aus bayerischem Anbau zur Verfügung standen. Die sogenannten Hilfs- und Melde-Arzneien kamen grundsätzlich aus China und waren nicht aus bayerischer Herkunft verfügbar. Die Arzneidrogen aus bayrischem Anbau wurden im Rahmen des Pflanzenbauprojekts von der Firma PhytoLab GmbH \& Co. KG (Vestenbergsgreuth, Deutschland) auf Inhaltsstoffe, Identität und Reinheit überprüft; die chinesische Handelsware (Plantasia - Großhandel für asiatische Heilkräuter, Oberndorf, Österreich) war bezüglich Reinheit und Identität zertifziert. Voruntersuchungen hatten ergeben, dass die Arzneidrogen aus China und Bayern von den Inhaltsstoffmustern wie auch von den Mengen vergleichbar waren [4].

Das Dekokt wurde von einer Apotheke standardisiert und konzentriert in Flaschen nach einem identischen Protokoll hergestellt sowie konserviert und nummeriert. Die Präparate waren optisch nur durch die Nummern unterscheidbar (Kasten 2).

Die Dekokte wurden an die einzelnen Studienärzte abgegeben, die diese dann an die Patienten im 2-wöchigen Rhythmus aushändigten. Der Arzt entschied völlig frei, ob zum Basisrezept ergänzend weitere Arzneimittel erforderlich waren und ob er diese zusätzlich als Dekokt, Granulat oder Fertigmedikament verordnete. Jede weitere Therapie war erlaubt (Akupunktur, Naturheilverfahren, schulmedizinische Medikation, Homöopathika), musste aber umfassend dokumentiert werden. Bezüglich der Herkunft (ob aus bayerischem oder chinesischem Anbau) sollten ursprünglich die Ärzte wählen können, allerdings wurden die Zuordnungsnummern erst am Studienende von der liefernden Apotheke mitgeteilt, sodass bezüglich der Provenienz der Dekokte eine Post-hoc-Verblindung stattfand.

Voraussetzung für die Teilnahme waren Volljährigkeit, klare Diagnose einer chronischen Rhinosinusitis nach den Leitlinien der Deutschen Gesellschaft für Hals-Nasen-Ohren-Heilkunde, Kopf- und Hals-Chirurgie [11], eine chinesische Syndromdiagnose und eine Beschwerdestärke von mindestens 4 auf einer numerischen Rating-Skala von 0 bis 10. Ausschlusskriterien waren eine bestehende Schwangerschaft, eine Polyposis nasi, Nikotinkonsum, eine medikamentöse Immunsuppression sowie eine fehlende Einverständniserklärung.

Die Patienten wurden gebeten, vor Behandlungsbeginn einen Einverständnisbogen sowie 2 und 4 Wochen nach Behandlungsbeginn einen Fragebogen auszufüllen. Vier Wochen nach Ende der Therapiebeobachtung wurden die Patienten erneut telefonisch von der Studienmitarbeiterin befragt. Der Patientenbogen enthielt Fragen zum Allgemeinzustand («Wie ging es Ihnen in den letzten 2 Wochen»), zu den Hauptsymptomen (laufende Nase, verstopfte Nase, Geruchsverlust, Infektneigung, Kälte-ZugluftAversion, spontanes Schwitzen) und den Allgemeinsymptomen (Appetit, Stuhlverhalten), die auf numerischen Rating-Skalen von -5 bis +5 bzw. von 0 bis 10 beantwortet werden sollten. Im Bogen zu Behandlungsbeginn wurden Alter, Geschlecht, Dauer der Beschwerden und bisherige Behandlungsversuche abgefragt. Im Verlaufsbogen, der nach 2, 4 und 8 Wochen ausgefüllt werden sollte, wurden die Patienten außerdem zum Einnahmeverhalten, zum Therapieerfolg und gegebenenfalls zu unerwünschten Wirkungen befragt.

Die beteiligten Ärzte dokumentierten bei Behandlungsbeginn sowie nach 2 und 4 Wochen die Indikation, die Syndromdiagnose (mit Ranking der Relevanz) sowie alle weiteren Diagnosen und parallel dazu angewandte Therapien (Akupunktur, andere ergänzende Phytotherapeutika, Homöopathika, Allopathika). Bei der Verlaufsbeobachtung war zu dokumentieren, ob, und wenn ja, welche unerwünschten Wirkungen mit welchem Schweregrad auftraten und ob eine Kausalität plausibel war. Therapieerfolg und Compliance wurden auf einer numerischen Rating-Skala von 0 bis 10 bewertet. Wurde die Behandlung oder die Beobachtung vom Patienten oder vom Arzt abgebrochen, wurde dies nach Möglichkeit mit Angabe von Gründen dokumentiert. Die Datenerfassung wurde auf die Richtigkeit und Vollständigkeit durch die Studienmitarbeiterin bei Abgabe der Bögen kontrolliert, überprüft und ggf. durch Nachfragen beim Arzt oder Patienten berichtigt.

\section{Anleitung zur Herstellung des Dekokt-Konzentrats}

Teemischung für 14 Tagesdosen auswiegen (Tagesdosen $\times 14$ )

Großer Topf (10 l), Grobsieb (z.B. Teesieb, feinmaschiges Seiher, Gaze-Sieb), Feinsieb (z.B. Kaffeefilter), Trichter, 1 dunkle Glasflasche $1000 \mathrm{ml}$ mit Twist-off-Deckel, Thermometer

Glasflasche und Verschlüsse säubern

Drogenmischung mit 21 siedendem Wasser aufgießen und den Ansatz 3-4 h zugedeckt ziehen lassen. Anschließend offen zum Sieden bringen und nach Erreichen des Siedepunkts mindestens 20 min leicht köcheln lassen, den Prozess beenden, ein wenig ankühlen lassen und den noch heißen Sud über das Grobsieb und anschließend über ein Feinsieb abseihen

- Nach Reduktion auf 750 ml Dekokt-Konzentrat in die Glasflasche abfüllen und verschließen

- $\quad 0,75 \mathrm{ml}$ PHB-Ester-Verbindung $(0,1 \%)$ herstellen (25 Teile Propyl4-hydroxybenzoat (Nipasol ${ }^{\circ}$ ) und 75 Teile Methyl-4-hydroxybenzoat $\left(\right.$ Nipagin $\left.^{\circ}\right)$ ) und in Glasflasche einrühren, Flaschen drehen und nach vollständigem Abkühlen bis zur Verwendung kühl und lichtgeschützt lagern (maximale Haltbarkeit 1 Jahr)

- Zur täglichen Verwendung $50 \mathrm{ml}$ des Extraktes im Messbecher abmessen und mit $450 \mathrm{ml}$ heißem Wasser (mindestens $90{ }^{\circ} \mathrm{C}$ ) in Thermoskanne abfüllen.

Die erhobenen Daten wurden in pseudonymisierter Form in eine elektronische Datenbank (FileMaker Pro Advanced) eingegeben, in eine Excel-Datei umgewandelt und statistisch ausgewertet (mit R 3.1.1 GUI). Einzelne Variablen wurden im Rahmen der Dateneingabe in Kategorien zusammengefasst bzw. bei Zutreffen als ja bzw. nein in «Freitexte», «begleitende schulmedizinische Behandlung (ja/nein)» und «vorangegangene Behandlungen (ja/nein)» eingeteilt:

Altersklassen wurden gemäß persönlicher Angaben eingeteilt und der Body Mass Index (BMI) wurde berechnet.

Zur Qualitätssicherung erfolgten neben der doppelten Dateneingabe Plausibilitätschecks. Die Datenauswertung erfolgte anhand des Datensatzes vom 15.07.2014 mit Angaben von gesamt 64 Patienten (Gruppe China - chinesische Kräuter: 33 Patienten; Gruppe Bayern - chinesische Kräuter aus bayerischem Anbau: 31 Patienten).

Initial wurden die demografischen Daten sowie die Daten zur Diagnosestellung beider Gruppen vor Beginn der Behandlung verglichen und mithilfe eines zweiseitigen t-Tests für unverbundene Stichproben auf einem Signifikanzniveau von 5\% getestet. Binäre Angaben bzw. Häufigkeiten wurden mit dem Test nach Fisher ebenfalls auf einem Signifikanzniveau von 5\% auf ihre Vergleichbarkeit hin geprüft.

Für die Analyse der Wirksamkeitsdaten wurden die Angaben der Patienten zur Stärke ihrer Symptombeschwerden sowohl auf signifikante Unterschiede zwischen den Gruppen zu den unterschiedlichen Zeitpunkten als auch die Veränderung der Symptome im Zeitverlauf zu den Zeitpunkten «vor Behandlung», «Woche 2», «Woche 4» und «Woche 8» untersucht. Da es sich um ordinale Daten handelt, wurden nicht-parametrische Tests angewandt. Um Unterschiede der Symptombewertung zwischen den Gruppen zu analysieren, wurde der Wilcoxon-Rang-Summentest für alle Erhebungszeitpunkte auf einem Signifikanzniveau von 5\% durchgeführt. Mit dem gleichen Test und dem gleichen Signifikanzniveau wurde die Veränderung der Symptome im Zeitverlauf geprüft. Insgesamt wurden 64 Patienten nach Intention-to-treat-Kriterien aufgenommen, nach der Per-protocol-Analyse waren es 61 Teilnehmer. Im Folgenden werden für alle Daten der Mittelwert, das 95\%-Konfidenzintervall (95\%KI) und der Median dargestellt. 


\section{Ergebnisse}

\section{Patientenfluss und Auswertungskollektive}

Insgesamt wurden 65 Patienten für die Therapiebeobachtung von den beteiligten Praxen rekrutiert. Davon wurde vor Beginn der

Tab. 1. Demografische Daten der Studienteilnehmer

\begin{tabular}{llll}
\hline & Gesamt & China & Bayern \\
\hline Anzahl, n (\%) & $64(100)$ & $33(52)$ & $31(48)$ \\
Alter in Jahren, MW (SD) & $45(13,1)$ & $45(14,8)$ & $45(11,2)$ \\
Weiblich, \% & $42(66)$ & $19(58)$ & $23(74)$ \\
BMI, kg/m2 & $24,1(3,5)$ & $23,8(2,9)$ & $24,3(4,1)$ \\
\hline
\end{tabular}

Dekokt-Zuteilung ein Patient ausgeschlossen, da er nicht den Einund Ausschlusskriterien entsprach. Von 64 Patienten mit Einnahme des Dekoktes brachen 2 Patienten die Studie innerhalb der ersten 2 Wochen der Behandlung ab; ein Patient wegen der Einnahme von Antibiotika, ein weiterer wegen Depression. Ein Patient brach die Studie in der 3. Woche (18. Tag nach Therapiebeginn) der Behandlung aufgrund von Nebenwirkungen ab (Abb. 1).

\section{Demografische Daten}

Mit Ausnahme eines stärkeren Anteils von 42,4\% Männern in der China-Gruppe im Vergleich zu 25,8\% in der Bayern-Gruppe sind die beiden Studiengruppen hinsichtlich demografischer Daten vergleichbar (Tab. 1).

Tab. 2. Chinesische Diagnosen

\begin{tabular}{lcccc}
\hline Syndromdiagnose, $\mathrm{n}(\%)$ & Gesamt, $\mathrm{N}=64$ & China, $\mathrm{n}=33$ & Bayern, $\mathrm{n}=31$ & $\mathrm{p}$-Wert \\
\hline $\begin{array}{l}\text { Algor, algor venti } \\
\text { Ja }\end{array}$ & $28(43,8)$ & $16(48,5)$ & $12(38,7)$ & 0,4607 \\
$\quad$ Nein & $36(56,3)$ & $17(51,5)$ & $19(61,3)$ & \\
Calor, calor venti & & & & 1,0000 \\
$\quad$ Ja & $35(54,7)$ & $18(54,5)$ & $17(54,8)$ & \\
$\quad$ Nein & $29(45,3)$ & $15(45,5)$ & $14(45,2)$ & \\
Humor/pituita & & & & 1,0000 \\
$\quad$ Ja & $58(90,6)$ & $30(90,9)$ & $28(90,3)$ & \\
$\quad$ Nein & $6(9,4)$ & $3(9,1)$ & $3(9,7)$ & \\
Depletio qi defensivum/ & & & & 0,2076 \\
$\quad$ pulmonalis & & & & \\
Ja & $52(81,3)$ & $29(87,9)$ & $23(74,2)$ & \\
$\quad$ Nein & $12(18,8)$ & $4(12,1)$ & $8(25,8)$ & \\
\hline
\end{tabular}

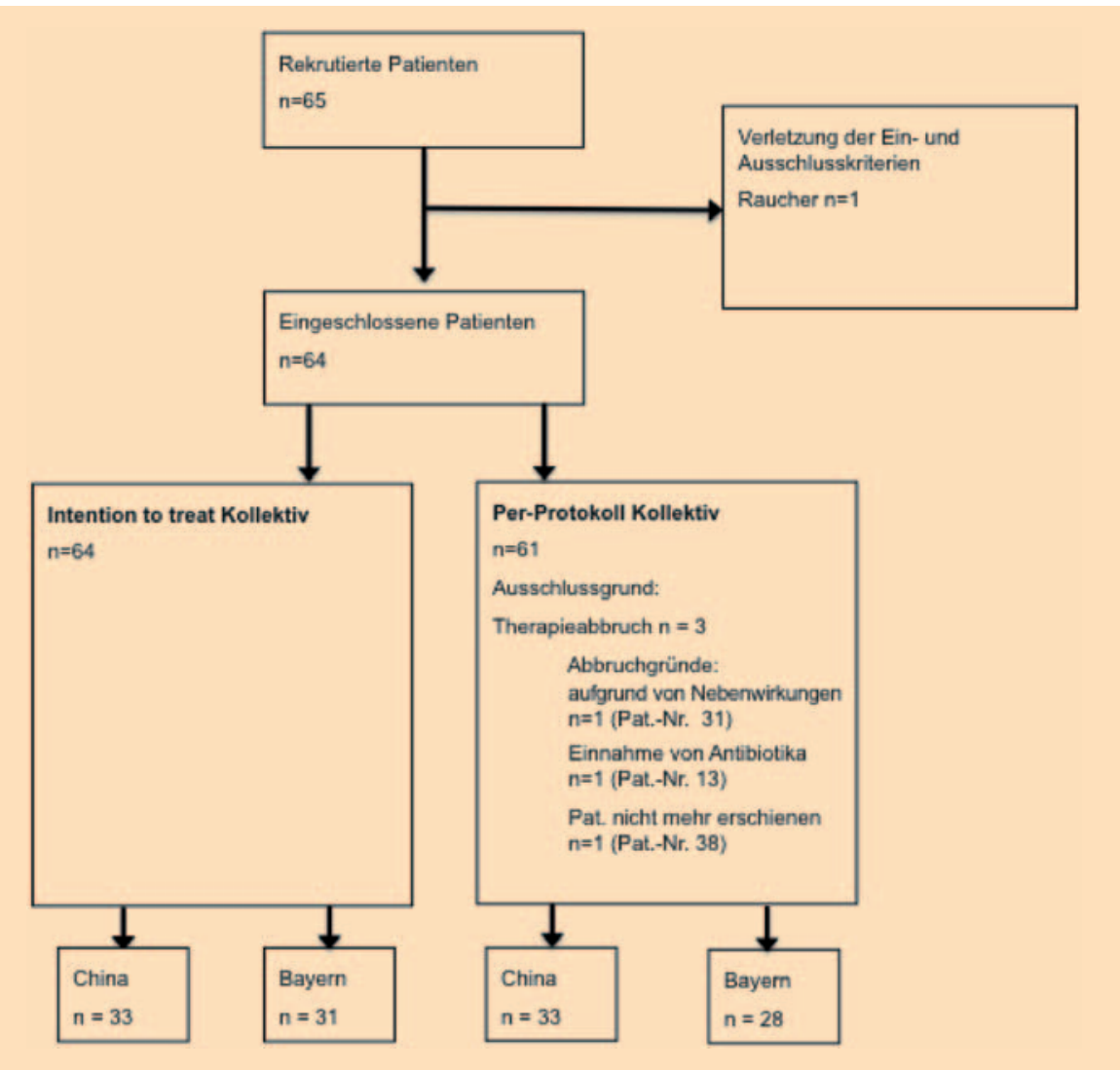

teilung der Patienten des Patientenflusses und AufPer-protocol-Kollektiv. 
Beide Gruppen wurden hinsichtlich der westlichen und chinesischen Diagnosen zu Studienbeginn verglichen (Tab. 2). Daraus ergaben sich in den Diagnosen und wesentlichen Erkrankungen keine signifikanten Unterschiede zwischen den Gruppen.

\section{Wirksamkeitsvergleich (Symptome im Zeitverlauf)}

Tabelle 3 zeigt die Ergebnisse der Bewertung der einzelnen Symptome zu den Zeitpunkten «vor Behandlung», «Woche 4» und «Woche 8». Dabei werden nur Symptome aufgeführt, die für die untersuchten Krankheitsbilder relevant sind wie «spontanes Schwitzen», «Empfindlichkeit gegen Kälte und/oder Zugluft», «Infektneigung», «verstopfte Nase», «Geruchsverlust» und «laufende Nase».

Die Veränderungen der Symptombewertungen wurden mit dem Wilcoxon-Test auf Signifikanz getestet.

Insgesamt zeigten sich keine Unterschiede in der Wirksamkeit zwischen den beiden untersuchten Gruppen. Im Beobachtungszeitraum zeigten sich aber für die Hauptsymptome und auch den Allgemeinzustand in beiden Gruppen eine deutliche Besserung (Abb. 2; Abb. 3). Das in der TCM wichtige Symptom der Verschleimung an unterschiedlichen Lokalisationen, besonders im Schleimhautbereich des Respirationstrakts, nahm über die Dauer der Behandlung in beiden Gruppen ab (Abb. 4).

\section{Beurteilung der Wirksamkeit der Therapie aus Sicht des Arztes}

Neben der Beurteilung der Wirksamkeit der Therapie wurde im Untersuchungsbogen für den behandelnden Arzt auch die Veränderung der Erkrankung bzw. der Beschwerden des Patienten abgefragt. Diese war überwiegend positiv.

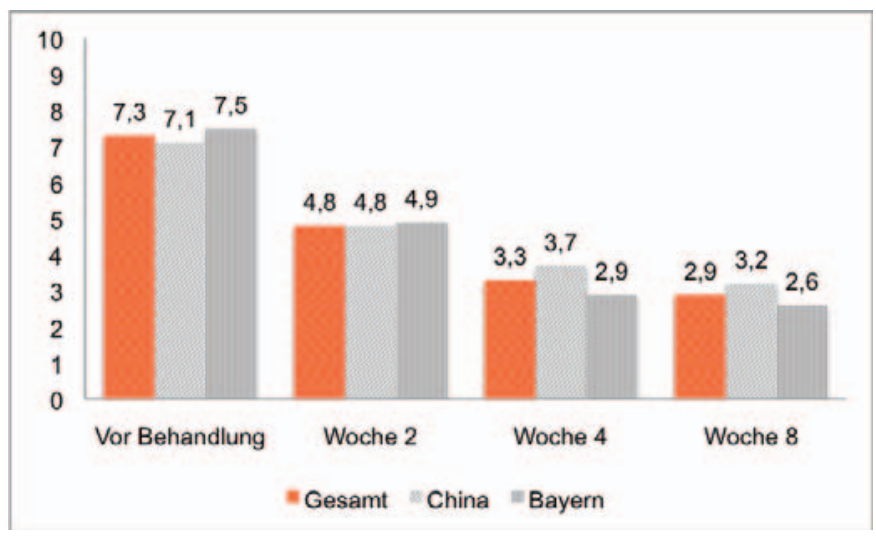

Abb. 2. Bewertung der Symptomstärke «verstopfte Nase» durch die Patienten auf einer numerischen Rating-Skala von 0 (überhaupt nicht) bis 10 (sehr stark).

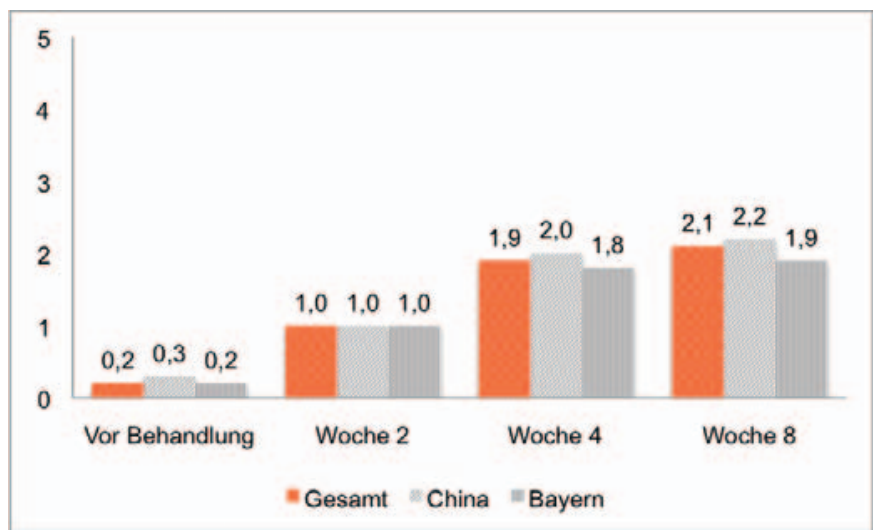

Abb. 3. Einschätzung des allgemeinen Gesundheitszustands durch die Patienten.
Tab. 3. Bewertung der einzelnen Symptome durch die Patienten in den RatingSkalen (Bereich 0-10)

\begin{tabular}{|c|c|c|c|c|c|c|c|}
\hline \multirow{2}{*}{ Zeitpunkt } & \multicolumn{3}{|c|}{ Gruppe China } & \multicolumn{3}{|c|}{ Gruppe Bayern } & \multirow{2}{*}{ p-Wert } \\
\hline & $\mathrm{n}$ & Median & $95 \%-\mathrm{KI}$ & $\mathrm{n}$ & Median & $95 \%-\mathrm{KI}$ & \\
\hline \multicolumn{8}{|l|}{ Spontanes Schwitzen } \\
\hline Vor Behandlung & 33 & 3 & $2,49-4,78^{*}$ & 30 & 4 & $2,71-5,09$ & 0,73 \\
\hline Woche 4 & 32 & 1 & $1,09-2,91^{*}$ & 29 & 1 & $1,39-3,65$ & 0,58 \\
\hline Woche 8 & 32 & 0 & $0,42-1,83^{*}$ & 29 & 1 & $1,34-3,21$ & 0,04 \\
\hline \multicolumn{8}{|c|}{ Empfindlichkeit gegen } \\
\hline \multicolumn{8}{|c|}{ Kälte und/oder Zugluft } \\
\hline Vor Behandlung & 33 & 7 & $5,01-7,35^{\star}$ & 30 & 8 & $6,13-8,27$ & 0,18 \\
\hline Woche 4 & 31 & 4 & $2,67-5,01^{*}$ & 29 & 6 & $3,44-6,07$ & 0,25 \\
\hline Woche 8 & 32 & 4 & $2,56-4,81$ & 29 & 3 & $2,98-5,43$ & 0,59 \\
\hline \multicolumn{8}{|l|}{ Infektneigung } \\
\hline Vor Behandlung & 33 & 6 & $4,67-6,96^{*}$ & 30 & 7,5 & $5,71-7,89$ & 0,21 \\
\hline Woche 4 & 29 & 5 & $3,36-6,02^{*}$ & 27 & 5 & $3,01-5,8$ & 0,82 \\
\hline Woche 8 & 32 & 2 & $1,79-4,15^{\star}$ & 29 & 4 & $2,87-5,27$ & 0,13 \\
\hline \multicolumn{8}{|l|}{ Verstopfte Nase } \\
\hline Vor Behandlung & 33 & 7 & $6,37-7,88^{*}$ & 30 & 8 & $6,84-8,29$ & 0,34 \\
\hline Woche 4 & 32 & 3 & $2,53-4,78^{*}$ & 29 & 2 & $1,83-3,75$ & 0,26 \\
\hline Woche 8 & 32 & 1,5 & $1,97-4,41^{*}$ & 29 & 2 & $1,47-3,64$ & 0,64 \\
\hline \multicolumn{8}{|l|}{ Laufende Nase } \\
\hline Vor Behandlung & 33 & 6 & $4,59-6,32^{*}$ & 30 & 5 & $3,77-5,63$ & 0,2353 \\
\hline Woche 4 & 32 & 1 & $1,43-3,63^{*}$ & 29 & 1 & $1,06-3,15$ & 0,5837 \\
\hline Woche 8 & 32 & 0,5 & $1,11-3,33^{*}$ & 29 & 0 & $0,92-2,94$ & 0,8341 \\
\hline
\end{tabular}

Hummelsberger et al. 
Tab. 4. Anzahl der Patienten mit «sehr gutem» oder «gutem» Erfolg nach 4 Wochen (Zusammenführung der Fragen zur Wirksamkeit aus Sicht des Arztes und aus Sicht des Patienten)

\begin{tabular}{|c|c|c|c|c|c|}
\hline \multirow{11}{*}{ 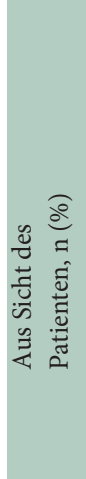 } & \multirow{6}{*}{$\cong$} & & \multicolumn{2}{|c|}{ Aus Sicht des Arztes, n (\%) } & \multirow[b]{2}{*}{ Gesamt } \\
\hline & & & $\mathrm{Ja}$ & Nein & \\
\hline & & Gesamt & $30(49)$ & $3(5)$ & $33(54)$ \\
\hline & & China & $15(45)$ & $2(6)$ & $17(51)$ \\
\hline & & Bayern & $15(54)$ & $1(4)$ & $16(57)$ \\
\hline & & Gesamt & $10(16)$ & $18(30)$ & $28(46)$ \\
\hline & \multirow{5}{*}{ 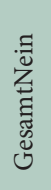 } & China & $5(15)$ & $11(33)$ & $16(49)$ \\
\hline & & Bayern & $5(18)$ & $7(25)$ & $12(43)$ \\
\hline & & Gesamt & $40(66)$ & $21(34)$ & $61(100)$ \\
\hline & & China & $20(61)$ & $13(39)$ & $33(100)$ \\
\hline & & Bayern & $20(71)$ & $8(29)$ & $28(100)$ \\
\hline
\end{tabular}

Tab. 5. Unerwünschte Ereignisse gemäß Patientenangaben

\begin{tabular}{lccc}
\hline & Gesamt, $\mathrm{N}=62$ & China, $\mathrm{n}=33$ & Bayern, $\mathrm{n}=29$ \\
\hline Unerwünschte Ereignisse, $\mathrm{n}$ (\%) & & & \\
Keine unerwünschten Ereignisse & $54(87,1)$ & $28(84,8)$ & $26(89,7)$ \\
Blähungen & $1(1,6)$ & $1(3,0)$ & $0(0)$ \\
Durchfall, Hypertonie & $1(1,6)$ & $0(0)$ & $1(3,4)$ \\
Magenbeschwerden & $1(1,6)$ & $0(0)$ & $1(3,4)$ \\
Magenschmerzen nach Dekokteinnahme & $1(1,6)$ & $1(3,0)$ & $0(0)$ \\
Müdigkeit - nach Teeeinnahme sehr schläfrig & $1(1,6)$ & $1(3,0)$ & $0(0)$ \\
Nase macht bei den Tropfen etwas «zu» & $1(1,6)$ & $1(3,0)$ & $0(0)$ \\
Übersäuerung des Magens/ Reflux & $1(1,6)$ & $1(3,0)$ & $0(0)$ \\
Verschlechterung 1 Tag nach Einnahme (Dauer 1-2 Tage) & $1(1,6)$ & 0 & $1(1,6)$ \\
\hline
\end{tabular}

Tab. 6. Parallel zur Dekokt-Behandlung eingesetzte komplementäre und schulmedizinische Behandlungen

\begin{tabular}{lccc}
\hline $\begin{array}{l}\text { Andere, parallel eingesetzte kom- } \\
\text { plementäre Behandlungsmethoden, } \mathrm{n}(\%)\end{array}$ & Gesamt, $\mathrm{N}=61$ & China, $\mathrm{n}=33$ & Bayern, $\mathrm{n}=28$ \\
\hline Keine & $18(29,5)$ & $8(24,2)$ & $10(35,7)$ \\
Akupunktur & $40(65,6)$ & $24(72,7)$ & $16(57,1)$ \\
Phytotherapie & $14(23,0)$ & $9(27,3)$ & $5(17,9)$ \\
Homöopathie & $5(8,2)$ & $4(12,1)$ & $1(3,6)$ \\
\hline
\end{tabular}

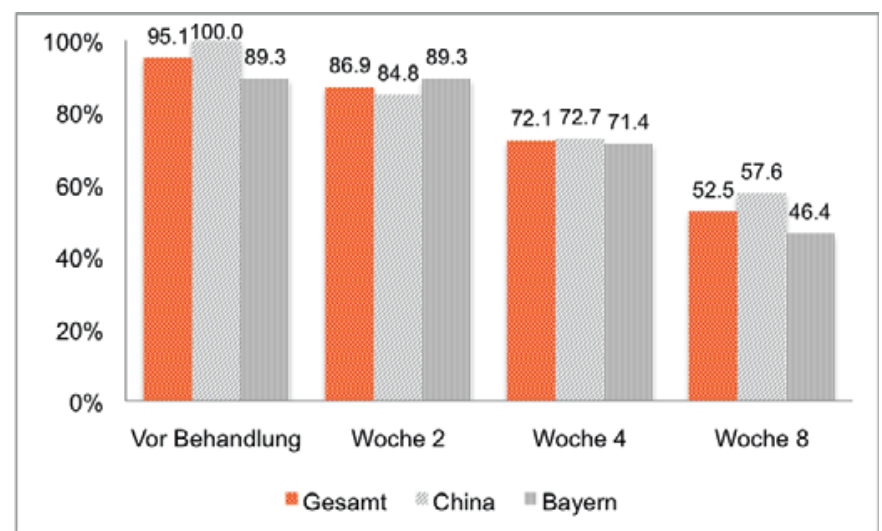

Abb. 4. Prozentualer Anteil der Patienten mit Verschleimung der Nase $(0=$ keine Verschleimung bis $10=$ starke Verschleimung $)$.

\section{Unterschiede in der Beurteilung der Wirksamkeit durch Ärzte und Patienten}

Tabelle 4 zeigt die zusammengefassten Anteile der Patienten mit «sehr guter» und «guter» Wirksamkeit bzw. «viel besserer» und «besserer» Veränderung des Krankheitsbildes, bewertet durch den Arzt und den Patienten zu den Zeitpunkten 2 und 4 Wochen bzw.
4 und 8 Wochen. Die Ergebnisse zeigen, dass die Patienten in Woche 4 die Wirksamkeit im Vergleich zu den behandelnden Ärzten kritischer beurteilten.

Unerwünschte Ereignisse und Beurteilung der Verträglichkeit

Tabelle 5 stellt die unerwünschten Ereignisse dar. Hier unterscheiden sich die beiden Gruppen nicht; auch traten keine schweren unerwünschten Ereignisse auf, sodass die Verträglichkeit insgesamt als gut beurteilt werden kann.

Leichte unerwünschte Ereignisse traten häufiger in der ChinaGruppe als in der Bayern-Gruppe auf. Zusammenfassend zeigt sich jedoch, dass die Dekokt-Behandlung sowohl von der Mehrzahl der Patienten als auch von den Ärzten als «sehr gut» bis «gut» beurteilt wurde.

\section{Zusätzliche Behandlungsformen}

Nur bei 7 der 61 Patienten wurde das Dekokt in Woche 2 modifiziert (3 Bayern-Gruppe, 4 China-Gruppe) und bei 54 unverändert über die gesamte Beobachtungszeit verabreicht.

Bei ca. $66 \%$ wurde parallel zur Dekokt-Behandlung Akupunktur eingesetzt; ca. 23\% erhielten ergänzend westliche bzw. chinesische 
Phytotherapeutika, 5 Patienten zusätzlich Homöopathika. In der Gesamtheit gab es hier keine Unterschiede zwischen den Gruppen. In der China-Gruppe wurde häufiger Akupunktur angewendet, in der Bayern-Gruppe wurden Phytotherapeutika eingesetzt (Tab. 6).

\section{Diskussion}

Wichtig für die Anwender und Apotheker ist das Ergebnis, dass sich in der Einschätzung durch den Patienten oder den behandelnden Arzt keine klinisch relevanten Unterschiede in der Wirksamkeit zwischen dem Dekokt aus original chinesischen Kräutern und dem in Bayern hergestellten Dekokt ergaben: beide Dekokte waren mehrheitlich gut verträglich, sowohl in der Beurteilung der Patienten als auch in der des Arztes. Zudem gab es keine Unterschiede in der Häufigkeit und Schwere von unerwünschten Therapiewirkungen.

Analysiert man die Verbesserung der relevanten Symptome im Zeitverlauf, so zeigen sich für die Mehrzahl der Symptome im Zeitraum zwischen 4 bzw. 8 Wochen klinische Verbesserungen. Vier Wochen nach Beendigung der Dekokt-Einnahme konnten jedoch bis auf die Symptome «Infektneigung», «spontanes Schwitzen» und «Empfindlichkeit gegen Kälte und/oder Zugluft» keine messbaren Verbesserungen mehr gezeigt werden.

Die Aussagekraft der Studie insgesamt ist aus methodischen Gründen deutlich eingeschränkt. Stärken der Studie sind die große Praxisnähe, eine sehr geringe Drop-out-Rate sowie das Faktum, dass durch die verspätete Ausgabe der Dekokt-Codierung durch die Apotheke sowohl Arzt als auch Patient gegenüber der Herkunft der Arzneikräuter post hoc verblindet waren. Die Auswertung zeigt weiter, dass die Dekokte per se meist unverändert gegeben wurden. Allerdings ist es durch die parallele Behandlung mit anderen komplementären Verfahren (meist Akupunktur) und das besondere Setting nicht möglich, den Therapieerfolg eindeutig der Therapie mit den Arzneimitteln oder der Begleittherapie zuzuordnen. Um die statistisch belegte Äquivalenz zweier Therapien nachzuweisen, müsste ein anderes Studiendesign mit entsprechenden statistischen Analysen und größerer Fallzahl gewählt werden. Eine Beobachtungsstudie stellt aber einen adäquaten Ansatz für einen pragmatischen Test auf Unterschiede dar. Auch wenn für die hier vorgestellte Studie keine formelle Äquivalenzprüfung durchgeführt wurde, sprechen die Ergebnisse gegen klinisch relevante Wirksamkeitsunterschiede zwischen chinesischen Arzneimitteldrogen bayerischer und chinesischer Herkunft.

Zur Behandlung der allergischen Rhinitis mit Akupunktur und chinesischen Arzneimitteln wurde 2004 eine randomisierte kont- rollierte Studie durchgeführt [12], die eine deutliche Überlegenheit der Verum-Therapie gegenüber einer Sham-Behandlung nach 6 Wochen zeigte. In dieser Untersuchung wurde eine ähnliche chinesische Rezeptur wie im Fall der vorliegenden Studie angewendet, in mehreren Folgestudien wurde nur die Akupunkturwirkung weiter untersucht [13]. Damals schien der Effekt von Akupunktur und chinesischem Dekokt äquivalent zu sein, was auch für die aktuelle Therapiebeobachtung angenommen werden muss.

Zusammenfassend ist festzustellen, dass nach den Ergebnissen dieser Therapiebeobachtung und trotz der großen methodologischen Einschränkungen chinesische Arzneimittel aus bayerischem Anbau klinisch ähnlich wirksam erscheinen wie Arzneikräuter aus China. Unter der 4-wöchigen Studienmedikation kam es zusammen mit den Begleittherapien zu einem Symptomrückgang. Diese Pilotstudie sollte Anlass zur weiteren Forschung geben.

\section{Förderung}

Diese Studie wurde durch das Bayerische Staatsministerium für Ernährung, Landwirtschaft und Forsten sowie die Internationale Gesellschaft für Chinesische Medizin (SMS) gefördert. Sponsor ist die Gesellschaft für die Dokumentation von Erfahrungsmaterial der Chinesischen Arzneitherapie (DECA) $\mathrm{mbH}$, Wasserburg-Reitmehring.

\section{Dank}

Wir danken den Förderern und Sponsoren (Bayerisches Staatsministerium für Ernährung, Landwirtschaft und Forsten, SMS Internationale Gesellschaft für Chinesische Medizin, DECA gGmbH), allen teilnehmenden Studienärzten (Dres. Beier, Bourgeois, Fintelmann, Nögel, Neumüller-Steger, Rausch, Scheuermann, Wullinger, v. Hasselbach) für ihre Mitarbeit, Hannah Mentz (Studienmitarbeiterin) für die sorgfältige Datenerhebung, den beteiligten Projektpartnern (Prof. Bomme, Dr. Torres-Londoño, Firma Kräuter-Mix und PhytoLab) und den Mitarbeitern und Mitarbeiterinnen aller Praxen.

Prof. Klaus Lindes Hilfe war in der Erstellung des Studienplans, des Ethikantrags und des Manuskripts von unschätzbarem Wert. Auch Prof. Benno Brinkhaus und Prof. Claudia Witt haben uns beratend bei entscheidenden Fragen geholfen.

Unser ganz besonderer Dank gilt Prof. Ralph Kohnen, der die Studie ideell großartig unterstützt und gefördert hat, jedoch tragisch und viel $\mathrm{zu}$ früh Anfang März 2014 verstarb.

\section{Disclosure Statement}

Die Autoren erklären hiermit, dass keine Interessenskonflikte in Bezug auf dieses Manuskript bestehen.

\section{Literatur}

1 Härtel U, Volger E: Inanspruchnahme und Akzeptanz klassischer Naturheilverfahren und alternativer Heilmethoden in Deutschland - Ergebnisse einer repräsentativen Bevölkerungsstudie. Forsch Komplementärmed Klass Naturheilkd 2004;11:327-334.
2 Zhao ZZ, Hu Y, Liang ZT, et al.: Authentification is fundamental for standardization of Chinese medicine. Planta Med 2006;72:865-874.

3 Hempen N, Huber R: Qualität und Sicherheit chinesischer Arzneidrogen in Deutschland - ein Update. Forsch Komplementmed 2014;21:401-412.
4 Heuberger H, Bauer R, Friedl F, Heubl G, Hummelsberger J, Nögel R, Seidenberger R, Torres-Londoño P: Cultivation and breeding of Chinese medicinal plants in Germany. Planta Medica 2010:76; 1956-1962. 
5 Heuberger H, Bomme U, Friedmann B, Groß J, Kabelitz L, Reif K, Schmücker R, Torres-Londoño P: Drogenqualität chinesischer Heilpflanzen aus bayerischem Versuchsanbau im Vergleich zu Importware: Identität, sensorische Eigenschaften, Inhaltsstoffe und Reinheit. Chin Med 2008:23;119-135.

6 Wegener T, Schneider B; Working Party of the German Society of Phytotherapy: Proposals to enhance the quality of observational cohort studies. Phytomedicine 2003;10:700-707.

7 Schulz KF, Grimes DA: Case-control studies: research in reverse. Lancet 2002;359:431-434.
8 Appel B: Können industrieunabhängige Arzneimittelstudien unter der 12. AMG-Novelle noch durchgeführt werden? DZKF 2005; 1/2:48-55.

9 Kraft K, Loew D, Schneider B, et al.: Planung, Durchführung und Auswertung von Anwendungsbeobachtungen. Empfehlungen der Gesellschaft für Phytotherapie (GPHY). Arzneim-Forsch/Drug Res 1997;47: 990-994.

10 Lu YB: The treatment of sinusitis with sinusolve. J Chin Med 2002;69:57-62.
11 Leitlinie «Rhinosinusitis» Leitlinie der Deutschen Gesellschaft für Hals-Nasen-Ohren-Heilkunde, Kopfund Hals-Chirurgie: Langfassung 2011. www.awmf.org/ uploads/tx_szleitlinien/017-049l_S2k_Rhinosinusitis_ 2011-verl\%C3\%A4ngert.pdf (accessed 27.08.2015).

12 Brinkhaus B, Hummelsberger J, Kohnen R, et al.: Acupuncture and Chinese herbal medicine in the treatmen of patients with seasonal allergic rhinitis: a randomizedcontrolled clinical trial. Allergy 2004:59:953-960

13 Brinkhaus B, Ortiz M, Witt CM, et al.: Acupuncture in patients with seasonal allergic rhinitis: a randomized trial. Ann Intern Med 2013;158:225-234. 